\title{
Percepción del adolescente ante la conducta adictiva del padre de familia alcohólico
}

\section{Perception of the adolescent before the addictive behavior of the alcoholic parent Percepção do adolescente diante do comportamento obsessivo do pai alcoolista}

\section{Leticia Casique Casique, Teresita de Jesús Muñoz Torres ${ }^{2}$, Alicia Álvarez Aguirre ${ }^{1}$, Rosalba Alejandra Medina Silva ${ }^{3}$, Melissa Reyna Nava ${ }^{3}$}

${ }^{1}$ Doctora en Ciencias de Enfermería. Docente e investigadora del Departamento de Enfermería y Obstetricia División de Ciencias de La Salud e Ingeniería Campus Celaya Salvatierra (México). Correo electrónico: leticiacc_2004@yahoo.com.mx; alicia.alvarez@ugto.mx.

${ }^{2}$ Maestra en Ciencias de Enfermería. Profesor Hora-Clase del programa de Licenciatura en Enfermería de la Unidad Académica Multidisciplinaria Zona Media de la UASLP. Enfermera adscrita a los Servicios de Salud de San Luis Potosí, Jurisdicción Sanitaria No. IV (México). Correo electrónico: teresita._torres@hotmail.com.

${ }^{3}$ Estudiante de la Licenciatura en Enfermería y Obstetricia de la Universidad de Guanajuato Campus CelayaSalvatierra.

Cómo citar este artículo en edición digital: Casique Casique, L., Muñoz Torres, T.J., Álvarez Aguirre, A., Medina Silva, R.A. y Reyna Nava, M.

(2020). Percepción del adolescente ante la conducta adictiva del padre de familia alcohólico. Cultura de los Cuidados (Edición digital), 24 (56) Recuperado de http://dx.doi.org/10.14198/cuid.2020.56.11

Correspondencia: Av. Ing. Javier Barros Sierra No. 201 Esquina Av. Baja California. Ejido de Santa María del Refugio, Celaya Gto. C.P. 38110.

Correo electrónico de contacto: leticiacc_2004@yahoo.com.mx

Recibido:18/10/2019

Aceptado:23/01/2020

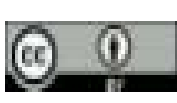

\section{ABSTRACT}

Objective: understand the adolescent's perception of the addictive behavior of the alcoholic parent. Methodology: qualitative and analytical research, carried out from September 2016 to May 2017, with N=12 adolescents. For the data collection, was used the semi-structured interview "Perception of the adolescent before the addictive behavior of the alcoholic father". The study procedure

included the application of an individualized interview, writing and analysis through the Grounded Theory of Data. Results: establishment of 4 categories through axial codes as a proper definition of the phenomenon of study and symbolic representation associated with the adolescent's perception of the addictive behavior of the alcoholic father. Conclusions: it can be interpreted that within the mexican culture the consumption of alcohol is allowed and well accepted, which 
fosters in young generations the conception that the consumption of alcohol is something "normal".

Keywords: addictive behavior, alcohol, perception, nursing.

\section{RESUMEN}

Objetivo: comprender la percepción del adolescente ante la conducta adictiva del padre de familia alcohólico. Metodología: investigación de tipo cualitativo y analítico, realizada de septiembre 2016-mayo 2017, con una $\mathrm{N}=12$ adolescentes. Para la recolecta de los datos se utilizó la entrevista semiestructurada "Percepción del adolescente ante la conducta adictiva del padre alcohólico". El procedimiento del estudio comprendió la aplicación de una entrevista individualizada, la redacción y análisis a través de la Teoría Fundamentada de los Datos. Resultados: establecimiento de 4 categorías a través de códigos axiales como una definición propia del fenómeno de estudio y representación simbólica asociada a la percepción del adolescente ante la conducta adictiva del padre alcohólico. Conclusiones: se puede interpretar que dentro de la cultura mexicana está permitido y bien aceptado el consumo de alcohol, lo que fomenta en las generaciones jóvenes la concepción de que el consumo de alcohol es algo "normal".

Palabras clave: conducta adictiva, alcohol, percepción, enfermería.

\section{RESUMO}

Objetivo: compreender a percepção do adolescente sobre o comportamento aditivo do pai alcoolista. Metodologia: Pesquisa qualitativa e analítica, realizada de setembro de 2016 a maio de 2017, com N = 12 adolescentes. Para a coleta de dados, utilizou-se 0 entrevista semiestruturada "Percepção do adolescente antes do comportamento aditivo do pai alcoolista". O procedimento do estudo incluiu a aplicação de entrevista individualizada, escrita e análise por meio da Grounded Theory of Data. Resultados: o estabelecimento de 4 categorias por meio de códigos axiais como definição adequada do fenômeno de estudo e representação simbólica associado à percepção do adolescente sobre o comportamento aditivo do pai alcoolista. Conclusões: pode-se interpretar que dentro da cultura mexicana o consumo de álcool é permitido e bem aceito, o que estimula nas gerações jovens a concepção de que o consumo de álcool é algo "normal".

Palavras-chave: comportamento aditivo, álcool, percepção, enfermagem.

\section{INTRODUCCIÓN}

Según la Organización Mundial de la Salud (2015), el alcohol es una sustancia psicoactiva con propiedades causantes de dependencia, que se ha utilizado ampliamente en muchas culturas durante siglos. El consumo nocivo de alcohol conlleva una pesada carga social y económica para las sociedades, por lo que es importante hacer consciencia a la sociedad sobre los daños que esta sustancia causa y el impacto sobre quienes la consumen.

Las estadísticas a nivel mundial mencionan que cada año ocurren 3.3 millones de muertes por consumo de alcohol, que representan un 5.9\% de defunciones, pues existe una relación causa-efecto entre el consumo de alcohol y los trastornos mentales o comportamentales, esto relacionado, a que el alcohol se ha considero una droga legal en comparación con otras sustancias (OMS, 2015); esta asociación causal, responde principalmente a su factor ambiental o social favorecido por las normas $\mathrm{y}$ leyes que hacen accesible el acceso y consumo del alcohol, incrementando la promoción al individualismo, los patrones 
Revista científica de la Asociación de Historia y Antropología de los Cuidados (Universidad de Alicante)

de consumo y el desarrollo de la de tal manera que el cerebro queda atrapado dependencia al alcohol, misma que ha en conductas que van en deterioro de su demostrado alterar la estructura $\mathrm{y}$ funcionamiento del Sistema Nervioso Central, provocando el evidente deterioro neuropsicológico (Ahumada, Gámez \& Valdez, 2017).

El consumo de alcohol y sus consecuencias a nivel mundial se basan en un historial sumamente largo relacionado con el consumo nocivo por parte de la sociedad y sus tradiciones (Fundación Alcohol y Sociedad, s.f.), la cual provoca efectos sanitarios y sociales perjudiciales para el bebedor, quienes lo rodean y la sociedad en general. El uso nocivo del alcohol puede comprometer al individuo alcohólico, no solo en lo personal arruinado su vida y la de su familia, sino también en lo social afectando a las personas que le rodean, así como en su salud, ya que desencadena diversas enfermedades crónicas que afectan la calidad de vida (Medline Plus, 2016).

En este énfasis, es importante señalar que la drogodependencia tiene connotaciones biológicas, psicológicas y sociales. En lo biológico, destaca la neuroplasticidad que supone la capacidad del sistema nervioso para adaptarse a condiciones ambientales que le permiten cambios en el comportamiento para la supervivencia, por medio de mecanismos como alteración del número de sinapsis, neurotransmisores disponibles, receptores postsinápticos, etc, adicción es entendida como una conducta habituada que ha ido ganando terreno al haber demostrado mayor capacidad porque existe una complejidad de factores de riesgo, vulnerabilidad y protección, vinculados no solo a un nivel neurobiológico, sino también psicológico y social. Por su parte, para el modelo sociocultural el consumo de sustancias es una interrelación cotidiana de experiencias subjetivas 0 sentidas en contextos naturales o espontáneos (Apud \& Romaní, 2016).

En cuanto a estadísticas en la población mexicana es importante destacar que la forma de consumo de alcohol típica en la población mexicana es de grandes cantidades y el consumo del mismo va en aumento, especialmente en adolescentes, los cuales imitan los patrones de consumo de alcohol de la población adulta. En México, una de cada tres personas de 12 a 65 años de edad mantiene un consumo nocivo de alcohol y al menos 500 mil son adictos a alguna sustancia ilegal (Cruz, 2015). Por su parte, la Encuesta Nacional de Consumo de Drogas, Alcohol y Tabaco 2016-2017 señala que el consumo en el hombre fue de $62.7 \%$ para el año 2016 con una dependencia de $3.9 \%$ y un promedio de ingesta de alcohol de 8.7 copas (Instituto Nacional de Psiquiatría Ramón de la Fuente Muñiz et al., 2017). 
En una investigación realizada en los

Centros de Integración Juvenil, se obtuvo que la mayor demanda de tratamiento fue por parte de los hombres (77.2\%), jóvenes de 15 a 19 años de edad (31.9\%), solteros (62.2\%) y con un nivel educativo de secundaria (45\%), 28.7\% eran estudiantes y el $25 \%$ tenían un trabajo estable, con un nivel socioeconómico medio-bajo (Comisión Nacional contra las Adicciones, 2011), lo cual demuestra que la edad, el nivel socioeconómico y la educación también son un factor importante para el inicio del consumo de sustancias nocivas para la salud, comenzando por el alcohol, el cual es más fácil y económico de conseguir en la mayor parte del país.

Por sus repercusiones sociales, económicas y morales sobre la familia y la sociedad, el alcoholismo se podría considerar la más grave de las adicciones, pues la ingesta está relacionada directa o indirectamente con más de cinco de las diez principales causas de defunción a nivel mundial y nacional (Juárez, 2014). Tan solo en la población mexicana el uso de alcohol es la cuarta causa de mortalidad (8.4\%), que implica cirrosis hepática, lesiones intencionales y no intencionales, accidentes de vehículo de motor y homicidios (Guerrero, Muñoz, Sáenz \& Reynaldes, 2012), los cuales provocan problemáticas sociales y familiares que repercuten considerablemente.

Es importante señalar que la educación es una parte primordial que lleva a percibir y realizar ciertas actividades que pueden ser importantes a lo largo de nuestra vida; sin embargo, no es una variable que por sí sola determine el inicio del consumo de alcohol en los adolescentes, ya que hoy en día se ve influenciado de forma importante por el ambiente que el adolescente percibe a su alrededor y sobre todo, la facilidad con la que el alcohol se puede conseguir (Assanelli, 2015).

La percepción del adolescente en el consumo de alcohol tiene gran influencia cuando los padres son o no consumidores de alcohol, ya que esa percepción es muy importante para que los adolescentes inicien el consumo del alcohol a edades tempranas y distingan esta conducta como algo bueno o malo, pues las actividades que los padres realizan, repercuten considerablemente en los hijos como un patrón repetitivo de la conducta de quien se supone es la máxima autoridad para ellos; aunque por otro lado, existen determinantes que de igual manera son importantes como factor de riesgo para el consumo del alcohol, como la permisividad por parte de los padres, la falta de comunicación, la violencia que estos puedan percibir, entre otros.

$\mathrm{Al}$ ser el consumo de alcohol un factor que afecta principalmente a los adolescentes y se asocia con la disponibilidad y características individuales que favorecen la probabilidad de experimentar, continuar usándolo, avanzar hacia el consumo problemático y dependencia, también se relaciona con estar expuesto al alcohol y tomar decisiones sobre 
Revista científica de la Asociación de Historia y Antropología de los Cuidados (Universidad de Alicante)

su conducta frente a la oportunidad (Assanelli, 2015; Gutiérrez et al, 2012).

De acuerdo al estudio realizado por Trujillo, Vázquez \& Córdova (2016) que tenía como objetivo identificar la percepción que tienen los adolescentes y sus padres sobre su funcionalidad familiar y su asociación con el consumo de alcohol, establecen que la percepción puede diferir en forma considerable desde el punto de vista del padre a la que percibe el hijo adolescente, pues es a este último, a quien afecta en forma directa los cambios físicos, psicológicos y sociales que ocurren. Este tipo de situaciones en las que el adolescente se ve reflejado puede implicar una situación arriesgada y puede afectar de manera considerable en el estilo de vida de forma negativa al sentir la necesidad de realizar actividades perjudiciales como el consumo de alcohol por el seguimiento de un patrón de la conducta (American Academy of Child \& Adolescent Psychiatry, 2015).

En este sentido, el Centro de Integración Juvenil (2016) del estado de Guanajuato declaro que el alcohol y tabaco registraron usos de 91\% (nacional: 86.2\%) y 89.3\% (nacional: 84.5\%) respectivamente, con una razón de consumo en el 2015 de 6.9 hombres por una mujer y con prevalencia en los grupos de 10 a 14 años (52.4\%) y de 15 a 19 años (37\%). Por su parte, los datos de la Encuesta Nacional de Consumo de Drogas, Alcohol y Tabaco 2016-2017 reportan en población masculina de 12 a 65 años un consumo diario de alcohol de $4.4 \%$ (media nacional: 4.5\%) y en población adolescentes de 12 a 17 años de 2.7\% (media nacional: 2.6\%) (Instituto Nacional de Psiquiatría Ramón de la Fuente Muñiz et al., 2017). Como es bien sabido el alcoholismo hoy en día es considerado como una enfermedad, porque afecta no solamente al consumidor de forma física, sino también en los distintos ambientes, ya sea de forma psicológica, económica, emocional y social (OMS \& OPS, 2015), por lo cual, se ha planteado como objetivo comprender la percepción del adolescente ante la conducta adictiva de su padre alcohólico, pues a través de la experiencia de quien vive el fenómeno, es que se pueden identificar los puntos de acción necesaria para mejorar la práctica del cuidado enfermero.

\section{METODOLOGÍA}

Investigación de tipo cualitativo y analítico, en la cual se realizó análisis de discurso y se aplicó la Teoría Fundamentada de los Datos. El estudio se realizó de septiembre 2016 a mayo 2017 con una muestra final de 12 adolescentes de la localidad de Los Laureles, Celaya, Gto. México; la selección de los sujetos fue de manera intencionada respetando los criterios de inclusión (adolescente entre 11 a 16 años según la Organización Mundial de la Salud, con progenitor consumidor de alcohol y que aceptara participar en el estudio firmando la anuencia correspondientes) y hasta obtener el muestreo teórico. Para la recolecta de los datos se utilizó la entrevista 
Cultura de los Cuidados

semiestructurada "Percepción del adolescente ante la conducta adictiva del padre alcohólico" el cual se divide en dos partes, en la primera parte se encuentran los datos personales de la entrevista y en la segunda, 4 preguntas que se enfocan en identificar a través del discurso la percepción del adolescente con respecto al consumo de alcohol por parte de su propio padre, es decir, su progenitor. El procedimiento del estudio comprendió la aplicación de la entrevista individualizada, llevada a cabo en las instalaciones de una institución educativa de la localidad y teniendo una duración de 30 minutos, una vez obtenida la información narrativa de los adolescentes se redactó lo obtenido, se realizó la triangulación de los datos y se analizó de manera artesanal, utilizando para tal fin, la Teoría Fundamentada de los Datos.

\section{RESULTADOS}

Se encontró que la distribución por grupo de edad mostró los siguientes valores, 33.3\% de los 11 a los 12 años de edad, el $50 \%$ entre los 13 y 14 años, y de 15 a 16 años con un $16.7 \%$, con relación al género, el 75\% fue femenino y $25 \%$ masculino. Dentro de sus ocupaciones extra-clases, solo el 25\% las realiza. Es importante resaltar, que de los participantes, 8.3\% se encontraban en unión libre al momento del estudio y el 75\% practicaba la religión católica. Del total de participante, $83.3 \%$ vive con sus padres y/o hermanos mientras que el $16.7 \%$ menciona la convivencia exclusiva con su madre. En el aspecto de proveedor económico, el 75\% de los adolescentes señaló que tal acción es responsabilidad de ambos padres.

En relación a los datos cualitativos en base a la Teoría Fundamentada de los Datos, se elaboró un mapa conceptual donde se describe el origen de las categorías a partir de la identificación de los códigos vivos, los cuales se agruparon en códigos axiales según denominados comunes para poder establecer juicios que permitan el analizar de estos y el establecimiento de categorías para cada pregunta (Fig. No.1). Para profundizar en el análisis se elaboró un mapa conceptual (Fig. No. 2) para establecer la definición de las categorías correspondientes al fenómeno de estudio. Por último, la figura 3 describe la representación simbólica asociada a la percepción del adolescente ante la conducta adictiva del padre alcohólico.

\section{DISCUSIÓN}

La percepción que tiene el adolescente ante la conducta adictiva de su padre alcohólico es una situación que afecta en la conducta presente y futuro de los mismos, por lo cual es considerada como una problemática sobre el tema del consumo de alcohol. En México, al tener una elevada incidencia entre los jóvenes adolescentes, es un factor de riesgo para la salud de la población que lo consume y por lo tanto una problemática para la salud pública, ya que conlleva consecuencias físicas y mentales que afectan la calidad de vida de las familias mexicanas y la sociedad en general.

Méndez et al (2015) mencionan que la población que más consume alcohol son los 
Revista científica de la Asociación de Historia y Antropología de los Cuidados (Universidad de Alicante)

adolescentes entre 12 a 19 años de edad y conforme pasa el tiempo el consumo de alcohol va aumentando tanto en hombres como en mujeres, en los cuales se ha observado que el efecto que causa el alcohol depende en gran cantidad del sexo del progenitor y el hijo, es decir, que los hijos de sexo masculino cuya madre hayan tenido problemas con el alcohol son más propensos a consumir sustancias de este tipo que en las adolescentes, por lo que se reflexiona que los adolescentes buscan la socialización y la aceptación por parte de otros, sobre todo cuando aumenta la presión que ejercen los amigos y compañeros de clases, contexto social que los hace vulnerables para realizar conductas de riesgo.

Como primera categoría se encontraron conductas simbólicas de las familias mexicanas a través del consumo de drogas licitas, actividades lúdicas, motivos de reunión familiar, personas significativas y consumo de alimentos, en donde la población adolescente señala sobre las celebraciones de las familias mexicanas y su forma de festejarlas que en su mayoría están representadas por reuniones con personas significativas, como la familia o los amigos; por ejemplo, Lucia menciona "empiezan festejando y pues si es cumpleaños con pastel y así... y si es por juntarse nada más...pues hacen con carne asada y toman hasta el final”, por su parte, Pardini señala que "depende de lo que estén festejando, mi familia festeja con cerveza y con música”, esto indica que en la mayoría de las familias mexicanas se acostumbra celebrar los eventos sociales con alcohol, música y comida.

En un estudio realizado por Cantu (2016) señala que los jóvenes regiomontanos acostumbran celebrar en compañía de todos sus seres queridos como familia, amigos etc, mientras que Maita et al (2015) mencionan que la segunda causa de consumo de alcohol está asociada a las reuniones familiares, sin embargo, Suarez et al (2016) establecen que los adolescentes creen que la forma de beber de los adultos está determinada por la "tradición", ya que basan su conducta en aseverar que "se bebe igual que antes", "no es nuevo" y "siempre se ha bebido igual".

El patrón de consumo en las personas adultas se identifica con un modelo "tradicional", cuyos elementos fundamentales son la normalización de la bebida, la vinculación del consumo con diversión o actos de celebración y con el consumo abusivointensivo. En este sentido, los adolescentes consideran que su propia conducta de consumo se ajusta a este modelo, y también que la forma de ingerir alcohol en la etapa adolescente se ha mantenido estable a lo largo de los años (Suarez et al, 2016). Al compararlo los resultados de la presente investigación sé refleja que las tradiciones están muy presente a la hora de reunirse con otras personas y la mayoría de estas familias coinciden en la forma de festejo, es decir, la presencia de elementos como la música, el baile, la comida en general y las bebidas alcohólicas, por lo que los adolescentes lo 
Fig. 1 Origen de las categorías

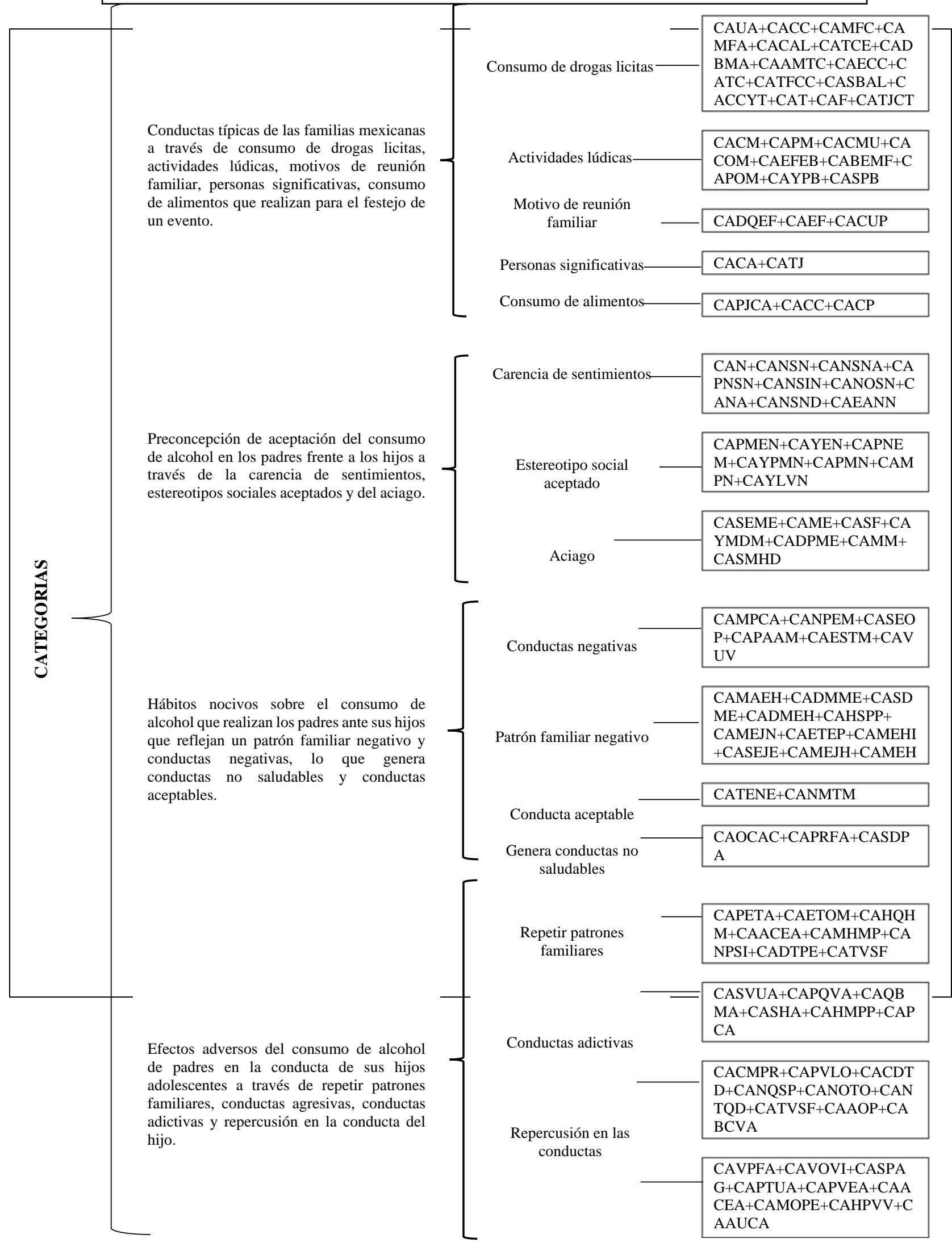

Fuente: Elaboración propia. (Apéndice 1) 
Figura 2. Mapa Conceptual

Representación simbólica de la conducta adictiva del padre en reuniones familiares, actividades lúdicas y festejo de eventos con una preconcepción de aceptación, presencia de patrones familiares negativos, conductas negativas y no saludables con repercusión negativa en los adolescentes

Conductas típicas de las familias mexicanas a través del consumo de drogas licitas, actividades lúdicas, motivos de reunión familiar, con personas significativas y consumo de alimentos que realizan para el festejo de un evento.

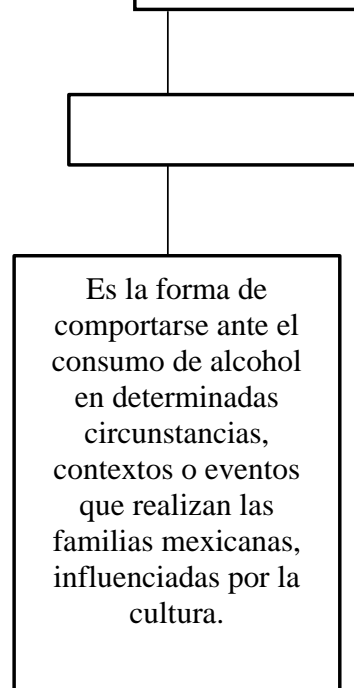
aceptación de consumo de alcohol en los padres frente a los hijos a través de la carencia de sentimientos, estereotipos sociales aceptados y del aciago.

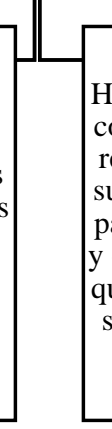

Hábitos nocivos sobre el consumo de alcohol que realizan los padres ante sus hijos que reflejan un patrón familiar negativo y conductas negativas, lo que genera conductas no saludables y conductas aceptables.
Efectos adversos del consumo de alcohol de padres en la conducta de sus hijos adolescentes a través de repetir patrones familiares, conductas agresivas, conductas adictivas y repercusión en la conducta del hijo.
Es la actitud de aceptación que tienen los adolescentes ante el consumo de alcohol de los padres de familia.

\section{DEFINICIONES}
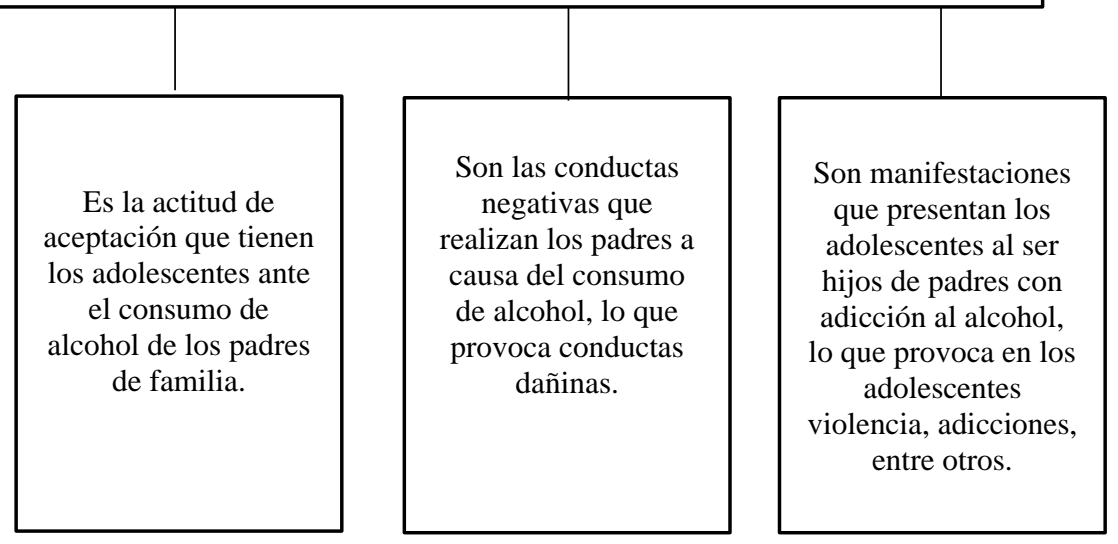

Fuente: Elaboración propia 
Fig. 3 Red conceptual de la percepción del adolescente ante la conducta adictiva del padre alcohólico

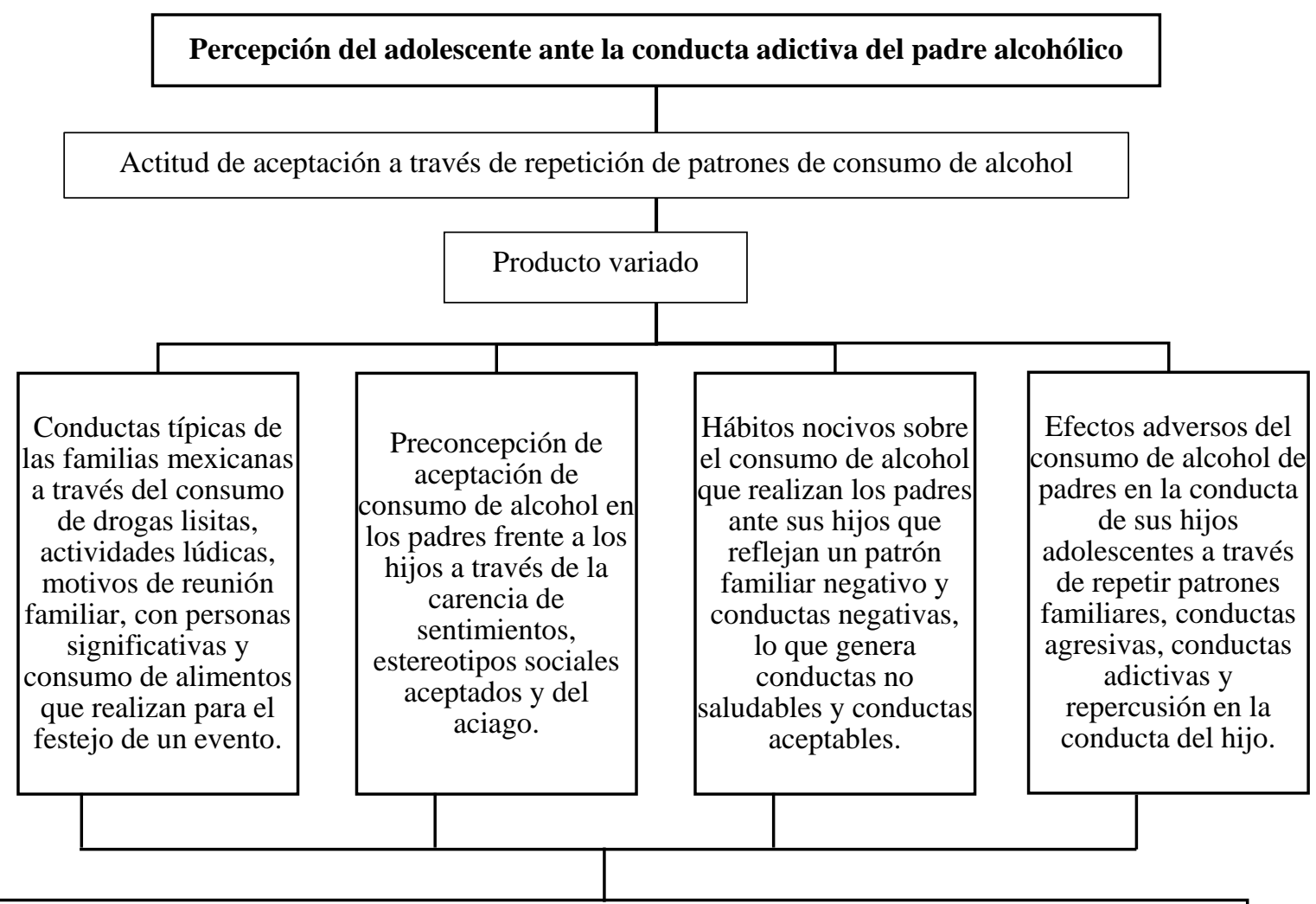

Dentro de la cultura mexicana está permitido y bien aceptado que, ante cualquier evento, ya sea de alegría, tristeza, coraje, etc. se tiene que acompañar con el consumo de alcohol, lo que fomenta en las generaciones jóvenes la idea de que todo festejo se DEBE de CELEBRAR consumiendo alcohol.

Representación simbólica de la conducta adictiva del padre en reuniones familiares, actividades lúdicas y festejo de eventos con una preconcepción de aceptación, presencia de patrones familiares negativos, conductas negativas y no saludables con repercusión negativa en los adolescentes.

Fuente: Elaboración propia 
Revista científica de la Asociación de Historia y Antropología de los Cuidados (Universidad de Alicante)

perciben como "normal” para la celebración de cualquier tipo de evento, al darse cuenta que la mayoría lo hace como una forma estereotipada de diversión.

La segunda categoría es preconcepción de aceptación del consumo de alcohol en los padres frente a sus hijos a través de la carencia de sentimientos, estereotipo social aceptado y aciago. En donde se vio que los adolescentes aceptan el consumo de alcohol por parte de sus padres como algo normal a comparación de cuando eran niños sentían decepción y tenían concepción sobre esas conductas, ya que mencionan estar acostumbrados al consumo de sus progenitores, como lo menciona Alexandra quien describe "a mí me parece normal, cuando era pequeña me enojaba y me molestaba...en la actualidad no siento nada”, pues al considerarlo como algo adecuado origina que la mayoría de las conductas de consumo sean aceptables, siempre y cuando el consumo no sea excesivo y más aún, si los padres de familia lo realizaban.

En un estudio realizado por Copello et al (2016) se identificó que la familia presenta una tendencia inversa en la apreciación, es decir, se observó que cuando las familias presentan algún grado de divergencia en la comunicación y adaptabilidad, pueden no tener adecuados estilos de afrontamientos que les permitan llegar a un acuerdo ante determinadas situaciones conflictivas, por lo que no son capaces de utilizar mecanismos estabilizadores que les proporcionen salud y bienestar a sus miembros así como protección ante conductas adictivas y las secuelas socio-familiares más significativas derivadas de la conducta del paciente alcohólico.

Otro estudio realizado por Suarez (s.f), señala que la conceptualización del alcohol bajo el prisma de droga institucionalizada, provoca que su consumo sea considerado socialmente como "normal" o "legítimo", y que los adultos sean más permisivos y benevolentes con los jóvenes que consumen alcohol, además con esta conducta, la sociedad adulta traslada de forma indirecta al adolescente la idea de ausencia de riesgo en el consumo de alcohol y puede que muchos adolescentes hayan desarrollado su propia idea acerca de cuál es el modelo social de consumo de alcohol en su grupo sociocultural de referencia, adaptándolo finalmente a sus propias necesidades hedónicas y de socialización.

En general, la familia está impregnada de una gran carga de angustia al no saber qué hacer ante el consumo y sus consecuencias, por ello, serán significativas las repercusiones de las adicciones desde el punto de vista individual, familiar y social, ya que un nivel de instrucción alto en una familia favorece el establecimiento de afrontamientos positivos ante la adicción pues se sabe que la educación de los padres influye positiva o negativamente en los hijos 
de acuerdo a como haya sido esta en cuanto a su conducta, modo de actuar y de pensar. La tercera categoría es hábitos nocivos sobre el consumo de alcohol que realizan los padres ante sus hijos que reflejan un patrón familiar negativo y conductas negativos, lo que genera conductas no saludables $\mathrm{y}$ conductas aceptables, en la cual se encontró que los adolescentes visualizan el consumo de alcohol de los padres como un mal ejemplo hacia los hijos, en este escenario Victoria describe "Yo pienso que está mal, siento que pueden ocasionar accidentes, pueden ocurrir problemas familiares, en el caso de los hijos que sigan los pasos de sus padres”.

Villareal et al (2013) encontraron que en el ámbito familiar, un funcionamiento familiar caracterizado por la vinculación emocional entre los miembros de la familia y la habilidad para adaptarse a diferentes situaciones y demandas de la dinámica familiar, se relacionan positivamente con el apoyo familiar. De igual manera los adolescentes estudiados ven esta actividad como algo "normal" tal como lo menciona Pardini “Pues en lo personal yo no pienso que este mal, pero tampoco pienso que este bien siempre y cuando no sea en exceso” y Alexandra "Pues yo pienso que no está mal si lo tomas con moderación, si se exceden pueden ocasionar problemas”. Musitu et al (2015) encontraron que los adolescentes que consumen alcohol con la familia y con los iguales se autoperciben como "personas sociales normales” e incluso se autoevalúan positivamente en los ámbitos personal y familiar y, lo más llamativo, es probablemente el sentimiento que tienen de que no están cometiendo ninguna infracción. Por lo que es importante mencionar que los adolescente se ven influenciados se manera importante por los padres de familia al percibir el consumo de alcohol como un hábito normal en las reuniones y en eventos sociales, lo cual es preocupante en la población adolescente mexicana, ya que en un futuro se verá influenciado por la toma de decisiones que afecten no solo a las familias mexicanas sino también a la sociedad en general, pues pueden estar presentes eventos de gran magnitud que puedan llegar a ocasionar hasta la muerte.

La cuarta y última categoría, es efectos adversos del consumo de alcohol de padres en la conducta de sus hijos adolescentes a través de repetir patrones familiares, conductas agresivas, conductas adictivas y repercusión en la conducta del hijo, en la cual, los participantes mencionan sobre la influencia que tiene el consumo de alcohol en los padres de familia sobre los hijos así como la conducta y las consecuencias que conlleva, pues el 100\% coincidió con que es un mal ejemplo que repercute al generar un patrón de conducta repetitivo ante las conductas adictivas que conllevan a consecuencias graves a nivel social $y$ familiar; en este punto, Victoria refiere "Pues se pueden volver violentos y causar más problemas también que como decía que beban más y se hagan alcohólicos” y Martha señala "Pueden empezar a tomar alcohol desde muy pequeños y así se vuelve 
Revista científica de la Asociación de Historia y Antropología de los Cuidados (Universidad de Alicante)

una adicción que es probable que se vuelvan alcohólicos, se pueden volver violentos al resolver algún problema con un familiar, compañero o amigo”. Por otro lado, también lo perciben como una forma de ejemplo para que los hijos no repitan la conducta de los padres ya que ellos lo deciden, como lo menciona Wendy, "Si deciden tomar es porque los papas lo enseño... y si no deciden tomar...es porque ellos quieren ser diferentes tener una vida más sana en su familia”.

El alcoholismo es común en las familias y los hijos de alcohólicos tienen una probabilidad cuatro veces mayor que otros de convertirse en alcohólicos. La mayoría de los hijos de padres alcohólicos han experimentado cierta forma de abandono o abuso; en estudios realizados, señalan que este tipo de familia puede tener una variedad de problemas como son Culpabilidad, Ansiedad, Temor y Vergüenza, por otro lado, como han sido decepcionados tantas veces por el padre/madre que bebe, no confía en otros, generando Confusión, Ira y Depresión (American Academy of Child and Adolescent, 2015).

\section{CONCLUSIONES}

Al realizar el análisis de discurso y aplicar la Teoría Fundamentada en los Datos se identificaron cuatro categorías, las cuales al ser analizadas de forma conjuntan permiten conocer el fenómeno, el cual es una representación simbólica de la conducta adictiva del padre en reuniones familiares, actividades lúdicas y festejo de eventos con una preconcepción de aceptación, presencia de patrones familiares negativos, conductas negativas y no saludables con repercusión negativa en los adolescentes; por lo que en base al análisis de dicho fenómeno, se puede interpretar que dentro de la cultura mexicana está permitido y bien aceptado que ante cualquier evento, este tiene que estar acompañado por el consumo de alcohol, lo que fomenta en las generaciones jóvenes la concepción de que el consumo de alcohol es algo "normal".

Es importante que el profesional de enfermería pueda influir en la reducción de la demanda de drogas, dado que es la ciencia del cuidado y que trabaja en la dirección del cambio y transformación de los estilos de vida, conductas y conciencia sanitaria de las personas, familias y grupos. Es así que los enfermeros deben de impulsar acciones en el campo de la promoción y educación en salud, ya que representan el mayor contingente de profesionales de la salud, pero sobre todo, son el primer contacto de la sociedad con los servicios de salud.

\section{BIBLIOGRAFÍA}

Ahumada, J.G., Gámez, M.E., \& Valdez, C. (2017). El consumo de alcohol como problema de salud pública. Ra Ximhai, 13 (2), 13-24.

American Academy of Child \& Adolescent Psychiatry. (2015). Los Hijos de Alcohólicos. Recuperado de http://www.aacap.org/AACAP/Families _and_Youth/Facts_for_Families/FFF- 
Spanish/Los-Hijos-de-Alcoholicos-

017.aspx.

Apud, I., \& Romaní, O. (2016). La encrucijada de la adicción. Distintos modelos en el estudio de la drogodependencia. Salud y drogas, 16 (2), 115-125.

Assanelli, N. (2015). ¿Qué Percepción tienen los Adolescentes Escolarizados entre 13 y 17 años de la ciudad de Florida sobre los riesgos Biológicos, Psicológicos y Sociales del Consumo de Alcohol?. Montevideo, Uruguay: Universidad de la Republica.

Cantú, B.O., De León, E., Pérez, D., \& Torres, M.S. (2016). Percepción que el adolescente residente de Monterrey mantiene sobre el regiomontano. Revista Electrónica en Iberoamérica Especializada en Comunicación, 20 (3), 1116-1136.

Centros de Integración Juvenil, A.C. (2016). Guanajuato. Recuperado de http://www.cij.gob.mx/patronatosCIJ/p df/Guanajuato.pdf.

Comisión Nacional contra las Adicciones. (2011). Programa contra el Alcoholismo y el Abuso de Bebidas Alcohólicas: Actualización 2011-2012. ( $1^{\text {ra }}$ Ed.). México: Secretaria de Salud. Recuperado de http://www.conadic.salud.gob.mx/pdfs/ publicaciones/abuso de bebidas.pdf.

Copello, O., et al. (2015). Vías conductoras a la adicción e intercambio con el medio familiar en adolescentes alcohólicos. Recuperado de http://www.medigraphic.com/pdfs/revh ospsihab/hph-2016/hph161e.pdf.

Cruz, A. (29 de enero del 2015). En México una de cada tres personas consume alcohol de manera nociva. La
Jornada.

Recuperado

de

https://www.jornada.com.mx/2015/01/2

9/sociedad/039n2soc.

Fundación Alcohol y Sociedad. (s.f). Hablemos de alcohol: por un nuevo paradigma del beber adolescente. Recuperado de http://www.alcoholysociedad.org/publi caciones/hablemos.aspx.

Guerrero, C.M., Muñoz, J.A., Sáenz, B., Reynaldes, L.M. (2012). Encuesta Nacional de Salud y Nutrición 2012. Evidencia para la política pública en salud. Consumo de alcohol en México 2000 - 2012: estrategias mundiales para reducir su uso nocivo. México: Instituto Nacional de salud pública. Recuperado de http://ensanut.insp.mx/doctos/analiticos /ConsumoAlcohol.pdf.

Gutiérrez, J.P., Rivera, J., Shamah, T., Villalpando-Hernández, S., Franco, A, Cuevas-Nasu, L., Romero-Martínez, M., Hernández-Ávila, M. (2012). Encuesta Nacional de Salud y Nutrición 2012. Resultados Nacionales. México: Instituto Nacional de Salud Pública. Recuperado de http://ensanut.insp.mx/informes/ENSA NUT2012ResultadosNacionales.pdf.

Instituto Nacional de Psiquiatría Ramón de la Fuente Muñiz, Instituto Nacional de Salud Pública, Comisión Nacional Contra las Adicciones, Secretaria de Salud. (2017). ENCODAT. Encuesta Nacional de Consumo de Drogas, Alcohol y Tabaco 2016-2017: Reporte de Alcohol. $1^{\text {ra }}$ Ed. México: Instituto Nacional de Psiquiatría Ramón de la Fuente Muñiz.

Juárez, V. (2014). Alcoholismo: Programa contra el alcoholismo y el abuso de sustancias alcohólicas. 
Revista científica de la Asociación de Historia y Antropología de los Cuidados (Universidad de Alicante)

México: Secretaria de salud del estado de Veracruz, Departamento de prevención y adicciones. Recuperado de http://web.ssaver.gob.mx/adicciones/pa gina-ejemplo/alcoholismo-2/.

Maita, M.V., Loja, A.L., \& Lupercio, R.N. (2015). Factores que influyen en el consumo de alcohol en adolescentes de 15 - 18 años de edad, de la unidad educativa fiscal "Francisco Febres Cordero". Cuenca, Ecuador: Universidad de Cuenca. Recuperado de http://dspace.ucuenca.edu.ec/bitstream/ $\underline{123456789 / 23254 / 1 / T E S I S . p d f}$.

Medline Plus. (2016). Riesgos del consumo de alcohol para la salud. A.D.A.M. Editorial. Recuperado de https://medlineplus.gov/spanish/ency/pa tientinstructions/000494.htm.

Méndez, M.D., Alonso, M.T., Alonso, M.M., Uribe, J.I., \& Armendáriz, N.A. (2015). Relación de percepción de riesgo y consumo de alcohol en adolescentes. Rev. Eletrônica Saúde Mental Álcool Drog, 11 (3), 161-167.

Musitu, G., Suárez, C., Del Moral, G., \& Villarreal, M.E. (2015). El consumo de alcohol en adolescentes: El rol de la comunicación, el funcionamiento familiar, la autoestima, el consumo en la familia y amigos. Rev. Búsqueda, 14, 45-61.

Organización Mundial de la Salud. (2015). Nota descriptiva $\quad N^{\circ} 349$. Recuperado de http://www.who.int/mediacentre/factsh eets/fs349/es/.

Organización Panamericana de la Salud y Organización Mundial de la Salud. (2015). Informe de situación regional sobre el alcohol y la salud en las Américas. Washington DC: OPS.

Suárez, C., Del Moral, G., Martínez, B., John, B., \& Musitu, G. (2016). El patrón de consumo de alcohol en adultos desde la perspectiva de los adolescentes. Gaceta Sanitaria, 30 (1), 11-17.

Suares, C., Mitsu, G., Vera, J.A., \& Ávila, M.E. (s.f.). Entre ciencia y experiencia: Un estudio cualitativo del consumo de alcohol en adolescentes. España: Universidad Pablo de Olavide Sevilla. Recuperado de https://rio.upo.es/xmlui/bitstream/handl e/10433/1182/cristian_suarez_tesis.pdf? sequence $=1$

Trujillo, T.T., Vázquez, E., \& Córdova, J.A. (2016). Percepción de la funcionalidad familiar y el consumo de alcohol en adolescentes. Rev. Aten Fam, 23 (3), 100-103.

Villarreal, M.A., Sánchez, J.C., \& Musitu, G. (2013). Análisis psicosocial del consumo de alcohol en adolescentes mexicanos. Universitas Psychologica, 12 (3), 857-873.

\section{APÉNDICE 1}

Lista de códigos

Consumo de drogas ilícitas

CAUA (código abierto utilizan alcohol) CACC (código abierto con cerveza)

CAMFC (código abierto mi familia con cerveza)

CAMFA (código abierto mi familia con alcohol)

CACAL (código abierto con alcohol)

CATCE (código abierto toman cerveza) CADBMA (código abierto diferentes bebidas mayoría alcohólicas)

CAAMTC (código abierto alcohol mayoría tequila cerveza) 
CAECC (código abierto empiezan con cerveza)

CATC (código abierto toman cerveza)

CATFCC (código abierto también festeja con cerveza)

CASBAL (código abierto siguen con bebidas aguas locas)

CACCYT (código abierto con cerveza y tequila)

CAT (código abierto toman)

CAF (código abierto fumar)

CATJCT (código abierto toman José cuervo tequila)

Actividades lúdicas

CACM (código abierto con música)

CAPM (código abierto ponen música)

CACMU (código abierto con música)

CACOM (código abierto con música)

CAEFEB (código abierto empiezan festejando evento baile)

CABEMF (código abierto bailan en mi familia)

CAPOM (código abierto ponen música)

CAYPB (código abierto y pues bailan)

CASPB (código abierto se ponen a bailar)

Motivo de reunión familiar

CADQEF (código abierto depende de lo que estén festejando)

CAEF (código abierto empiezan festejando)

CACUP (código abierto cumpleaños pastel)

Personas significativas

CACA (código abierto con amigos)

CATJ (código abierto todos juntos)

Consumo de alimentos

CAPJCA (código abierto por juntarse carne asada)

CACC (código abierto con comida)

CACP (código abierto con pastel)

Carencia de sentimientos

CAN (código abierto nada)
CANSN (código abierto no siento nada)

CANSNA (código abierto no siento nada)

CANSIN (código abierto no siento nada)

CAPNSN (código abierto personalmente no siento)

CANOSN (código abierto no siento nada)

CANA (código abierto nada)

CANSND (código abierto no siento nada)

CAEANN (código abierto en actualidad no siento nada)

\section{Estereotipo social aceptado}

CAPMEN (código abierto para mi es normal)

CAYEN (código abierto ya es normal)

CAPNEM (código abierto pues no está mal)

CAYPMN (código abierto ya para mi es normal)

CAPMN (código abierto para mi normal)

CAMPN (código abierto me parece normal)

CAYLVN (código abierto ya lo veo normal)

\section{Aciago}

CASEME (código abierto siento que es mal ejemplo)

CAME (código abierto me enoja)

CASF (código abierto siento feo)

CAYMDM (código abierto y me da miedo)

CAMM (código abierto me molesta)

CASMHD (código abierto si me han desilusionado)

Conducta negativa

CAMPCA (código abierto está mal que los papas consuman alcohol)

CANPEM (código abierto no pienso que este mal)

CASEOP (código abierto si exceden ocasionan problemas) 
Revista científica de la Asociación de Historia y Antropología de los Cuidados (Universidad de Alicante)

CAPAAM (código abierto papas agresivos con alcohol más)

CAESTM (código abierto está mal)

CAVUV (código abierto vuelvan violentos)

Patrón familiar negativo

CAMAEH (código abierto mal ejemplo hijos)

CADMME (código abierto dan muy mal ejemplo)

CASDME (código abierto si dan mal ejemplo)

CADMEH (código abierto dan mal ejemplo a los hijos)

CAMEJN (código abierto mal ejemplo para nosotros)

CAETEP (código abierto comienzan a tomar por el ejemplo de sus papas)

CAMEHI (código abierto mal ejemplo para los hijos)

CASEJE (código abierto sigan el ejemplo)

CAMEJH (código abierto mal ejemplo para los hijos)

CAMEH (código abierto mal ejemplo a hijos)

Conducta aceptable

CATENE (código abierto tampoco está bien no en exceso)

CANMTM (código abierto no está mal si lo tomas con moderación)

Genera conductas no saludables

CAOCAC (código abierto ocasionan accidentes)

CAPRFA (código abierto problemas familiares)

CASDPA (código abierto que sientan decepción por los papas)

Repetir patrones familiares

CAPETA (código abierto pueden empezar tomando alcohol)

CAETOM (código abierto ellos tomar)
CAHQHM (código abierto que los hijos quieran hacer lo mismo)

CAACEA (código abierto el alcohol causa efectos agresivos)

CAMHMP (código abierto cuando sean mayores hacer lo mismo que los padres)

CANPSI (código abierto negativa pueden ser iguales)

CADTPE (Código abierto si decide tomar es porque el papa los enseño)

CATVSF (código abierto tener una vida más sana en su familia)

\section{Conductas adictivas}

CASVUA (código abierto se vuelva una adicción)

CAPQVA (código abierto probable que se vuelvan alcohólicos)

CAQBMA (código abierto que beban más)

CASHA (código abierto se hagan alcohólicos)

CAHMPP (código abierto los hijos pueden seguir los mismos pasos)

Repercusión de las conductas

CACMPR (código abierto causar problemas)

CAPVLO (código abierto pueden volverse locos)

CACDTD (código abierto creer que deben tomar para divertirse)

CANQSP (código abierto no querer ser como sus papas)

CANOTO (código abierto no tomar)

CANTQD (código abierto no tomar por que ellos quieren ser diferentes)

CAAOP (código abierto afectar a otras personas)

CABCVA (código abierto bien por cómo van a actuar)

Conductas agresivas

CAVPFA (código abierto pueden volverse violentos al resolver problemas con familiares y amigos) 
Cultura de los Cuidados

CAVOVI (código abierto pueden volverse violentos)

CASPAG (código abierto se pongan agresivos)

CAPTUA (código abierto puedan tener un accidente)

CAPVEA (código abierto ponerse violentos por efecto del alcohol)

CAACEA (código abierto el alcohol causa efectos agresivos)

CAMOPE (código abierto pueden matar a otras personas)

CAHPVV (código abierto los hijos pueden volverse violentos)

CAAUCA (código abierto adoptan una conducta agresiva)

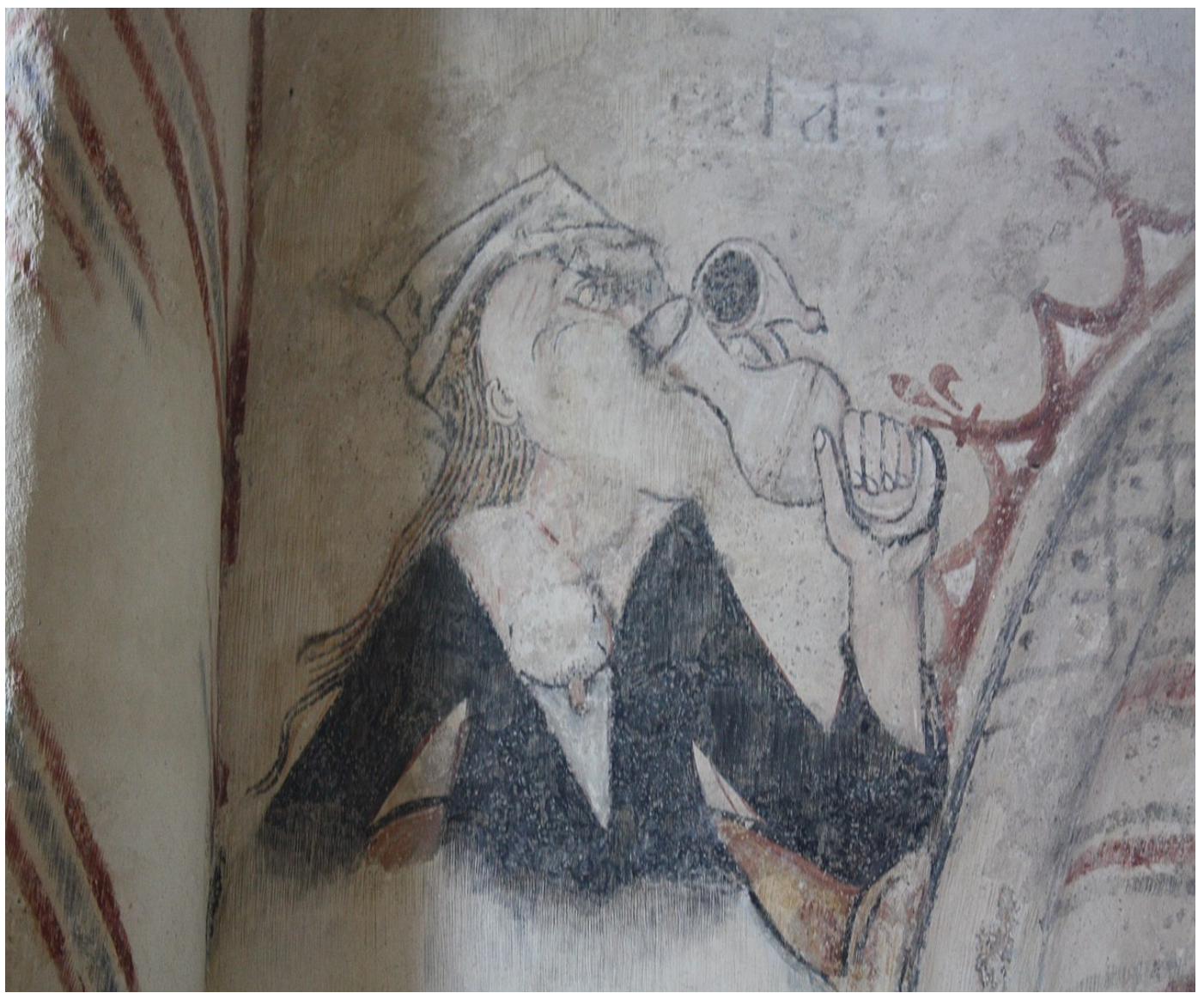

Fuente: A Gula, pinturas murais do Mosteiro de San Xulián de Moraime (Muxía, A Coruña). Unha muller bebe viño dunha xarra (Common Creative.Wikipedia). 